\title{
Intelligent Speed Regulation Control of Switched Reluctance Motor of Electric Vehicle Based on Neural Network Parameter Identification
}

\author{
Xiaoliang Zhang ${ }^{1}$ \\ ${ }^{1}$ Information Engineering School, Jiaozuo Normal College, Jiaozuo, Henan, China
}

\begin{abstract}
:
Based on the analysis of the related theories of switched reluctance motor, this paper designs and implements the intelligent speed regulation system of switched reluctance motor for electric vehicle. The speed regulation system has the characteristics of high efficiency and energy saving, wide speed regulation range and small starting current. The system also has the advantages of large starting torque, frequent start and stop, forward and reverse switching, high torque / inertia ratio and four quadrant operation. The system can provide a good solution for variable speed drive. The digital controller of the system is composed of TMS320F2812 DSP and EPM7128S CPLD, which can realize the starting, electric and braking functions of electric vehicles. Experiments show that the switched reluctance motor drive system for electric vehicle has good control performance and strong fault tolerance. The system can meet the requirements of various working conditions of electric vehicles and has broad development prospects.
\end{abstract}

Keywords: Neural Network, Parameter Identification, Electric Vehicle, Motor Intelligence, Speed Regulation Control.

\section{INTRODUCTION}

As the main tool of warehouse handling, forklift can replace the porters to do high-intensity handling work [1-2]. The flexibility and universality of forklift can effectively improve the efficiency of warehouse handling. At present, forklifts are mainly composed of manual forklifts, electric forklifts and internal combustion forklifts. The electric forklift consumes electric energy, which has alleviated the global energy crisis to a certain extent. Electric forklift belongs to the field of electric forklift and is a new energy project benefiting the country and the people [3]. At present, the main implementation form of electric forklift is DC electric forklift, which 
Article History: Received: 28 October 2021 Revised: 05 December 2021 Accepted: 10 January 2022 Publication: 28 February 2022

adopts DC motor control drive system [4]. The system determines the main performance of the electric forklift, the production efficiency of the whole vehicle and the safety performance of operation. With the continuous development of DC electric forklift, it will continue to expand its market share and provide users with electric forklift with better performance, higher efficiency, easier operation and higher safety performance. At the same time, the cost of electric forklift will continue to be reduced [5-6]. The continuous development and innovation of electric forklift is of great significance to China's automobile industry, environmental protection and national economic development [7]. The sustainable development of electric forklift will also have a far-reaching impact on the development of society.

\section{SLIDING MODE CONTROL AND SIMULATION OF SWITCHED RELUCTANCE MOTOR BASED ON REACHING RATE}

1.Sliding mode variable structure control

The difference between sliding mode variable structure control and other control methods lies in the discontinuity of control. It reflects the switching characteristics that the "structure" of the system changes according to time and needs [8-9]. The control characteristic can make "sliding mode" motion, even if the system moves with high flatness and small amplitude according to the predetermined state trajectory in a certain characteristic state. Since the sliding mode is independent of disturbance and system parameters, and can be designed according to needs, the system with sliding mode variable structure control has good robustness.

From a theoretical point of view, because the sliding mode motion of the system will not be affected by the parameter changes of the control object and external disturbances, the robustness of the sliding mode control system is better than that of the conventional control system. However, because the sliding mode variable structure control reflects the switching characteristics of the structure, which will cause the system Jitter. In the process of applying variable structure control to practice, the Jitter problem has become a prominent problem. Through analysis, it can be concluded that the main causes of Jitter are as follows [10]:

(1) Time lag switch. Near the switching surface, because of the time lag of the switch, the control function can not accurately follow the state change, but there is always a certain lag and delay for a certain time. At the same time, because the amplitude change of the control quantity follows the amplitude change of the state quantity, the binary value decreases gradually. Therefore, it shows that a decaying triangular wave is superimposed on the smooth sliding surface.

(2) Space lag switch. The spatial hysteresis of the switch is inevitable, and the spatial hysteresis will lead to the dead zone of the change of the state quantity in the state space. Therefore, it is shown that an equal amplitude waveform is superimposed on the sliding mode surface. 
Article History: Received: 28 October 2021 Revised: 05 December 2021 Accepted: 10 January 2022 Publication: 28 February 2022

(3) Influence of system inertia. Considering that the control ability of the system is limited, because the acceleration of the system can not be infinite and is limited. In addition, there is always inertia in the system, so there is always lag in the switching of the system. This lag caused by system inertia is equivalent to time lag.

(4) Jitter caused by discrete system structure. Considering the structural characteristics of the discrete system, its sliding mode is "quasi sliding mode", and its structure causes its switching action to occur on the surface of the cone with the fixed point as the origin, rather than on the switching surface. Therefore, it will lead to the jitter of attenuation, and the jitter amplitude is related to the conical volume.

2.Establishment of sliding mode variable structure control system

The motion process of sliding mode variable structure system consists of two parts. The first part is that the state space trajectory is outside the switching surface or crosses the switching surface a finite number of times; The second part is the sliding mode stage, in which the system moves along the switching surface near the switching surface.

From the characteristic knowledge of switched reluctance motor, it can be known that switched reluctance motor has the following three state equations:

$$
\begin{array}{r}
\frac{d i_{j}}{d t}=\frac{u_{J}}{L_{J}(\theta)}-\frac{r i_{j}}{L_{J}(\theta)}-\frac{d L_{J}(\theta)}{d \theta} \frac{i_{j}}{L_{J}(\theta)} \omega \\
\frac{d \omega}{d t}=\frac{1}{J}\left[\frac{1}{2} \sum_{J=1}^{m} \frac{d L_{J}(\theta)}{d \theta} i_{J}^{2}-B \omega-T_{L}\right] \\
\frac{d \theta}{d t}=\omega
\end{array}
$$

Where $u_{J}$ is the phase $j$ winding voltage, $i_{j}$ is the phase $j$ winding current, $L_{j}$ is the phase $j$ winding inductance, $r$ is the winding resistance, $B$ is the friction coefficient, $j$ is the moment of inertia, $\mathrm{T}_{\mathrm{L}}$ is the load torque, $\mathrm{w}$ is the rotational angular velocity, and $\theta$ is the rotor position angle.

According to the state equation of switched reluctance motor:

$$
\left[\begin{array}{c}
\dot{x}_{1} \\
\dot{x}_{2}
\end{array}\right]=\left[\begin{array}{cc}
0 & 1 \\
0-B / J &
\end{array}\right]\left[\begin{array}{l}
x_{1} \\
x_{2}
\end{array}\right]+\left[\begin{array}{l}
0 \\
-\frac{1}{J}
\end{array}\right] \dot{T}+\left[\begin{array}{l}
0 \\
\frac{1}{J} \dot{T}_{L}
\end{array}\right]
$$

If the load is considered to be constant and in the no-load state, equation (4) becomes:

$$
\left[\begin{array}{l}
\dot{x}_{1} \\
\dot{x}_{2}
\end{array}\right]=\left[\begin{array}{cc}
0 & 1 \\
O-B / J &
\end{array}\right]\left[\begin{array}{l}
x_{1} \\
x_{2}
\end{array}\right]+\left[\begin{array}{l}
0 \\
-\frac{1}{J}
\end{array}\right] \dot{T}
$$


Article History: Received: 28 October 2021 Revised: 05 December 2021 Accepted: 10 January 2022 Publication: 28 February 2022

Where $\mathrm{T}$ is the derivative of electromagnetic torque, take $\mathrm{T}$ as the output control quantity, i.e. $\mathrm{u}=\mathrm{T}$, then equation (5) becomes

$$
\left[\begin{array}{l}
\dot{x}_{1} \\
\dot{x}_{2}
\end{array}\right]=\left[\begin{array}{cc}
0 & I \\
0-B / J &
\end{array}\right]\left[\begin{array}{l}
x_{1} \\
x_{2}
\end{array}\right]+\left[\begin{array}{l}
0 \\
-\frac{1}{J}
\end{array}\right] u(6)
$$

The switching function is:

$$
s=s(x)=c x_{1}+x_{2}, c>0
$$

Then equation (8) holds.

$$
\dot{s}=\dot{s}(x)=c \dot{x}_{1}+\dot{x}_{2}=c x_{2}+\dot{x}_{2}
$$

\section{HARDWARE DESIGN OF SRD SYSTEM FOR ELECTRIC FORKLIFT}

\section{SRD system composition}

The SRD speed regulation system discussed in this paper is composed of four phase $8 / 6$ switched reluctance motor, main controller, photosensitive position sensor, power converter, current detection, voltage detection and so on. The photosensitive position sensor uses the position signal generated by rotor rotation to input the system controller after processing. The controller uses the feedback position signal as the basis for calculating speed, commutation and generating control drive signal. The current detection module provides a chopping signal for the current chopper by detecting the phase current on the winding, and also provides a trigger signal for the motor overcurrent protection. Voltage detection provides protection trigger signal for overvoltage and undervoltage protection of the system by detecting bus voltage. The control signals such as steering, speed and parking are given by the external analog signals, and the system calls the corresponding modules according to these control signals to realize the normal operation of SRD system.

2.Design of system main controller

System performance requirements: (1) speed accuracy. When the motor works under a certain load and the motor runs stably, the speed of the motor needs to be limited within a certain range centered on the given speed, that is, the steady-state error of the motor speed should be limited within a certain range. (2) Speed change rate. When the motor is running at a steady speed and the load changes suddenly, the change of motor speed should be within a certain range without large change or jitter. (3) Speed regulation range. The motor can speed within a certain speed range. The speed range of the motor should be determined according to the actual needs. The maximum speed of the system designed in this paper is 3000r / min according to the actual situation. 
Article History: Received: 28 October 2021 Revised: 05 December 2021 Accepted: 10 January 2022 Publication: 28 February 2022

The following protection functions are designed in the system: (1) undervoltage and overvoltage protection. By detecting the system bus voltage and comparing it with the preset voltage value, if the detected voltage is greater than or less than its corresponding set value, the system cuts off the power supply through the control signal to stop the system. (2) Overheating protection. The system converts the motor temperature into voltage value through thermistor, and compares it with the preset voltage value. If the temperature is higher than the protection value, the system cuts off the power supply and stops working. Overheating protection can avoid the damage of control system, power converter and motor itself due to high stability. It is one of the indispensable protection of the system. (3) Overcurrent protection. In order to avoid excessive current damaging power devices, the system adopts current protection measures. When excessive current is detected, the system automatically cuts off the power supply and stops working. (4) Overload protection function. When the electric forklift cannot be started successfully due to excessive load, the system will automatically stop working. The system can avoid the situation that the system can not operate under high current and high output torque for a long time, and can protect the system and motor from damage in time. (5) Position signal error protection function. The control drive signal of SRD system is based on the position signal. If the position signal is wrong, the control signal will also be wrong, resulting in irregular current applied to the motor and contradictory output torque, so that the system can not work normally. Through the detection and judgment of the position signal, if the position signal is found to be wrong, the system will stop the output of the control signal to stop the system. (6) The current sensor is faulty. Current detection is the precondition of current chopper control strategy and overcurrent protection. If the current detection is wrong, the system will not operate normally. If the current fault is detected, the system will stop working. (7) Overspeed protection. Considering the particularity of electric forklift as a means of transportation, for the sake of safety, its speed must be limited. When the motor speed is greater than a set value, the output of control signal will be stopped to stop the system.

\section{SOFTWARE DESIGN OF SRD SPEED REGULATION SYSTEM FOR ELECTRIC FORKLIFT}

The main program completes the system speed regulation function in the background. It mainly calls each calculation sub module to complete the relevant actions according to the corresponding control instructions. The main program in this system is mainly responsible for initializing the system, calling the starting sub module, giving the steering control system, fault detection and handling, etc. In the system, the setting of control commands, fault detection and resolution, and relevant control strategies are realized by calling the corresponding sub modules and relevant interrupt service sub modules.

DSP tms320lf2812 is a chip designed for controlling motor. Peripheral circuits such as 
Article History: Received: 28 October 2021 Revised: 05 December 2021 Accepted: 10 January 2022 Publication: 28 February 2022

event manager (EV), analog-to-digital conversion (AID) circuit and serial communication (SCI) are integrated in the chip. According to this characteristic of DSP, the hardware part of the system needs to be initialized before the system starts to work. At the same time, it is also necessary to initialize and set the relevant system working mode, parameters, control flag bit and interrupt system, so as to ensure that each functional module of DSP system can work according to the requirements. The initialization sub module is mainly used to initialize and set each part of the system according to the program requirements.

It mainly includes system clock setting, watchdog setting, initialization of used variables, I / $\mathrm{O}$ function port setting of DSP, setting of relevant flag bits in the system, etc. At the same time, various registers need to be initialized, such as setting the relevant control registers of timer unit, capture unit and full comparison unit of event manager, and setting the relevant control registers of aid, etc.

After the system is initialized, the relevant registers, control parameters and various functional modules in the system are configured correctly. At this time, the program enters the starting module according to the external input control command. When the program goes into the starting subroutine mode, the system first detects the external input instructions, determines the motor's steering according to the external input instructions, and then calls the corresponding positive or reverse functional modules. In the starting subroutine module, $1 / 2$ cycle full angle conduction is adopted, that is, all conduction is conducted in the rising region of the inductance. When the speed is low, the rotating electromotive force is small and the current rises rapidly. When $1 / 2$ cycle full angle conduction is adopted, the output torque of the motor can be as large as possible and the starting process of the motor can be accelerated.

From the previous analysis, it can be seen that when the motor is running at low speed, it is suitable to adopt current chopping control ((CCC)) mode because the current rises rapidly. At high speed, it is necessary to adjust the opening angle and closing angle to speed the motor. At this time, the system adopts angle position control (APC). This program judges which control strategy the system adopts according to the motor speed. In this procedure, in order to make the motor smoothly transition from low speed to high speed, hysteresis with hysteresis width of SOr/min is adopted. The angle position control (APC) mode is adopted when the motor is in the acceleration state and the rotating speed is greater than $800 \mathrm{r} / \mathrm{min}$. When the motor is running in a deceleration state, the current chopping control ((CCC)) mode will only be called when the rotating speed is less than $750 \mathrm{r} / \mathrm{min}$. In this way, the jitter effect of the motor when switching between the two control modes can be effectively avoided.

The capture interrupt subroutine first saves the capture value when the capture interrupt is generated. When a certain number is saved, the speed calculation subroutine module is called to calculate the motor rotor speed. Motor speed is not only a prerequisite for the selection of control mode and control mode, but also an indispensable control parameter for system speed regulation, which is very important to the whole system. The system first calculates the 
Article History: Received: 28 October 2021 Revised: 05 December 2021 Accepted: 10 January 2022 Publication: 28 February 2022

instantaneous speed of the motor rotor in the capture interruption, then calls the control mode to select the module, and the control mode selection module selects the control mode adopted by the motor according to the instantaneous value of the motor rotor. After determining the control mode, the system calls the PI speed regulation sub module to speed the motor. According to the output results of the PI algorithm module, combined with the commutation sub module, the corresponding control drive signal is given.

\section{CONCLUSION}

Switched reluctance motor is applied to electric forklift, which can realize the integration of electric and power generation of electric forklift. In the current situation of increasingly prominent environmental problems, it is worth our further study.

In this paper, the performance of switched reluctance motor is studied experimentally. Through the experiments of switched reluctance motor in starting, electric and braking States, the data and conclusions about the performance of switched reluctance motor with this structure are obtained. The optimization of electric angle position is studied. By calculating the speed and changing the opening angle and closing angle at different speeds, the goal of torque and efficiency optimization control is achieved. Through experiments, the feasibility of the proposed control strategy is verified, and the expected experimental results are obtained, which lays a foundation for further research in the future.

\section{ACKNOWLEDGEMENTS}

This research was supported by Science and Technology Planning Project of Henan Province, China (Grant No. 212400410223)

\section{REFERENCES}

[1]Lu Rui, Sun Linfu. Research on collaborative control method and software tool of distributed spare parts inventory for industrial chain cloud service platform. Computer Engineering and Science, 2017 (10): 36-42

[2]Pu Shiliang, Yuan Tingting. Discussion on intelligent service architecture of Internet of things based on cloud edge integration. Intelligent Internet of things technology, 2018, 1 (01): 7-12 [3]Lu Xiaoming, Xu Huayang, Shang Xiaodong, et al. Agent based collaborative framework for networked software resources. Command information system and technology, 2015, 6 (003): 3743 
Article History: Received: 28 October 2021 Revised: 05 December 2021 Accepted: 10 January 2022 Publication: 28 February 2022

[4]Xia Wei. Application of coal mine information collaborative management and control platform. Electronic technology and software engineering, 2018, 16: p.157-157

[5]Yu Yang, sun Linfu, Ma Yahua. Attribute based access control model for cloud manufacturing collaborative platform. Computer integrated manufacturing system, 2017, 23 (001): 196-202

[6]Lin Tong, Ma Jinfeng, Yang Huan, et al. Design and development of intelligent home drug management system based on cloud control. Computer knowledge and technology: academic exchange, 2018, V.14 (15): 83-85

[7]Hu Xiaona, Yang Peng, Liu Xuan. Content collaborative distribution mechanism based on broadcast storage structure. Computer applications and software, 2017 (10): 7-12 + 71

[8]Yuan yuan, promise. On the deployment of collaborative office portal for Disease Control and prevention based on the characteristics of cloud computing. Computer CD software and application, 2012, 5): 58-59

[9]Sun Liang. Building a hospital fine management and control system by using OA platform. Fujian computer, 2018, V.34 (06): 133-134

[10]Zhao Ruiqin, Shen Xiaohong, Zhang Xiaomin, et al. Research on the best relay model and broadcast mechanism of wireless multi hop network. Acta computer Sinica, 2014, 037 (002): 335-343 